\title{
Risk Factors Associated With Pouch Adenomas in Patients With Familial Adenomatous Polyposis
}

\author{
Petra Ganschow, M.D. ${ }^{1}$ Silke Trauth, M.D. ${ }^{2}$ Ulf Hinz, M.Sc. ${ }^{2}$ • Anja Schaible, M.D. ${ }^{2}$ \\ Markus W. Büchler, M.D. ${ }^{2}$ Martina Kadmon, M.D. ${ }^{3}$ \\ 1 Department of General, Visceral, Vascular and Transplantation Surgery, Ludwig-Maximilians University, Munich, Germany \\ 2 Department of General, Visceral and Transplantation Surgery, University of Heidelberg, Heidelberg, Germany \\ 3 School of Medicine, University of Augsburg, Augsburg, Germany
}

BACKGROUND: Restorative proctocolectomy with ileal pouch-anal reconstruction is the standard prophylactic surgical procedure for patients with familial adenomatous polyposis. However, several groups have reported the development of adenomas and even carcinomas within the ileal pouch. The predisposing factor was the time interval after pouch surgery in some studies, but it was the severity of the initial colonic disease and duodenal adenomatosis in others.

OBJECTIVE: The aim of this study was to further clarify the prevalence of pouch adenomas, clinical risk factors, and a possible phenotype-genotype relation in a large population of patients with familial adenomatous polyposis, as well as to analyze pouch adenoma-free survival.

DESIGN: This study was designed as a cohort study.

SETTINGS: This study was conducted in a specialized outpatient clinic at the University of Heidelberg.

PATIENTS: A total of 192 patients with familial adenomatous polyposis were included, and all of the available endoscopy

Supplemental digital content is available for this article. Direct URL citations appear in the printed text, and links to the digital files are provided in the HTML and PDF versions of this article on the journal's Web site (www.dcrjournal.com).

Funding/Support: None reported.

Financial Disclosure: None reported.

Preliminary results were presented at the meeting of the German Society for General and Visceral Surgery, Berlin, Germany, March 25 to 28, 2014.

Correspondence: Petra Ganschow, M.D., Department of General, Visceral, Vascular and Transplantation Surgery, Ludwig-Maximilians University, Marchionini-Str 15, München 81377, Germany. E-mail: petra. ganschow@med.uni-munechen.de

Dis Colon Rectum 2018; 61: 1096-1101

DOI: $10.1097 /$ DCR.0000000000001157

(c) The ASCRS 2018 reports after pouch surgery were screened for pouch adenomas. Additional clinical information was retrieved from the Heidelberg Polyposis Register.

MAIN OUTCOME MEASURES: This present study revealed 3 main independent risk factors for the development of pouch adenomas: age $<18$ years at the time of IPAA, male sex, and the presence of gastric adenomas. Secondary outcome measures were adenoma progression and overall pouch adenoma-free survival.

RESULTS: Pouch adenomas were detected in $46.9 \%$ of patients. Median follow-up was 12.8 years (interquartile range, 9.0-17.0 y) for patients with pouch adenomas and 7.3 years (interquartile range, 2.5-12.2 y) for those without them. Patients underwent pouch surgery at a median age of 27.5 years (range, 10.2-58.5 y), and pouch adenomas occurred a median of 8.5 years (range, 0.9-25.1 y) after surgery. Also detected were gastric adenomas in $37.2 \%$, duodenal adenomas in $80.3 \%$, and desmoid tumors in $24.5 \%$ of patients. Estimation of pouch adenoma-free survival revealed that, after 20 years, only $\approx 22 \%$ of patients would be free of pouch adenomas. Male sex, age $\leqq 18$ years at the time of pouch surgery, and gastric adenomas were found to be independent risk factors for the development of pouch adenomas in a multivariate Cox regression analysis $(p=0.0002$, $p=0.0059$, and $p=0.0020$ ). No predisposing germline mutation for pouch adenoma development was detected.

LIMITATIONS: Detailed information on the initial preoperative findings was not fully available, and the study was only carried out as a single-center study.

CONCLUSIONS: A severe upper intestinal phenotype, male sex, and age $<18$ years at the time of IPAA all increase the risk for development of pouch adenomas. See Video Abstract at http://links.lww.com/DCR/A675.

KEY WORDS: Familial adenomatous polyposis; Pouch adenomas; Pouch surgery. 
$\mathrm{P}$ atients with familial adenomatous polyposis (FAP) require prophylactic surgical intervention to prevent death from colorectal cancer. Colectomy followed by ileorectal anastomosis is the favorable therapeutic option for patients with FAP with little or no rectal involvement. In cases with rectal involvement, however, restorative proctocolectomy followed by IPAA, which restores good function and quality of life, is the procedure of choice. ${ }^{1-3}$

Prophylactic removal of the colon increases the life expectancy of patients with FAP, but extracolonic manifestations consisting mainly of desmoid tumors and duodenal adenomas and carcinomas gain in importance over the course of the disease. ${ }^{4}$ Several groups have also suggested that adenomas and carcinomas within the ileal pouch represent life-limiting factors. ${ }^{5-10}$ Reported rates of pouch adenomas vary between $35 \%$ and $57 \% .^{5-11}$ Data on risk factors are controversial, because they are based mainly on small patient series. Some studies have reported the time interval after pouch surgery and the severity of the initial colonic disease manifestation or duodenal adenomas to be predisposing factors, although others could not confirm these observations. ${ }^{5-10}$ To date, no relation between a germline mutation and pouch adenomas has been detected. ${ }^{8,9}$ The aim of the present study was to examine the prevalence and risk factors for the development of pouch adenomas in a large FAP patient sample. Secondary outcome measures were adenoma progression and overall pouch adenoma-free survival.

\section{PATIENTS AND METHODS}

The study was approved by the internal review board of the Heidelberg Medical Faculty. This study was designed as a cohort study. We identified 193 consecutive patients with FAP who had undergone pouch surgery and were followed up at our specialized outpatient clinic between May 2010 and August 2013. Data on follow-up were documented prospectively. At the beginning of the study period, a new standardized documentation sheet for pouch endoscopies was introduced (Supplemental Digital Content 1, http://links.lww.com/DCR/A743), which includes, among other details, a systematic description of pouch adenomas. Additional descriptive endoscopy reports were formulated for each pouch endoscopy.

The regular follow-up protocol included clinical examinations and yearly endoscopies of the pouch and of the pouch-anal anastomosis. The follow-up interval for upper GI endoscopies depended on the phenotype and was defined by the current Spigelman stage. ${ }^{12}$ In addition, abdominal ultrasounds and/or MRIs were performed if desmoid tumors were suspected and then repeated depending on findings and treatment.

All of the pouch endoscopy reports from the time of the patient IPAA $(n=1536)$ were reviewed for pouch adenomas and pouchitis. Likewise, all of the upper GI endoscopy reports were reviewed for gastric and duodenal adenomas. Additional clinical data, mutation analyses, and pathology reports were retrieved from clinical charts and the Heidelberg Polyposis Register. Adenomas arising from the remaining rectal mucosa were excluded from the analysis. The mean number of bowel movements per 24 hours was calculated based on the number of bowel movements at each follow-up appointment.

Led by the Spigelman classification, we defined pouch adenoma severity according to the number $(1-4,5-10$, or $>10$ adenomas) and size $(\leq 4,5-10$, or $\geq 10 \mathrm{~mm})$ of the adenomas. ${ }^{12}$ Progression of pouch adenomas was defined as an increase in the number and/or size of the adenomas, as well as a change in the histological pattern from tubular to villous or development of high-grade dysplasia or malignancy.

\section{Statistical Analysis}

SAS software (version 9.4, SAS Institute Inc, Cary, NC) was used for statistical analysis. The quantitative variables age at the time of IPAA, follow-up since IPAA, and number of bowel movements were expressed as the median with an interquartile range (IQR) or range. The nonparametric Mann-Whitney $U$ test was used to compare these quantitative variables between patients with and without pouch adenomas. Variables with a categorical score level are presented as absolute and relative frequencies. Subgroups of patients were analyzed using the Fisher exact test or the $\chi^{2}$ test, as appropriate. Adenoma-free pouch survival was defined as the time from the date of IPAA to either histological diagnosis of pouch adenoma or last follow-up. Patients without pouch adenomas at the last follow-up were censored. Follow-up time was given as the median with an interquartile range. Survival curves were calculated using the Kaplan-Meier method. The 5-, 10-, 15-, and 20-year pouch adenoma-free survival rates and the median survival time are presented. Differences in pouch adenoma-free survival between subgroups of patients were analyzed using the log-rank test. A multivariate Cox regression analysis was performed to assess the impact of variables on pouch adenoma-free survival. HRs with 95\% CIs are given. Two-sided values of $p<0.05$ were considered statistically significant.

\section{RESULTS}

A total of 193 patients were screened. Of those, 192 (100 males and 92 females) were enrolled in the study. One patient with an MUTYH mutation was excluded from the statistical analysis. Among all of the participants, the median follow-up after pouch surgery was 10.1 years (IQR, 5.5-15.4 y) and was 12.8 years (IQR, 9.0-17.0 y) among patients with pouch adenomas and 7.3 years (IQR, 2.5-12.2 y) among those without pouch adenomas. The median age at the time of IPAA was 
TABLE 1. Demographic, clinical, and histological data of study population $(\mathrm{N}=192)$

\begin{tabular}{lcc} 
Parameter & Median (IQR) & $n(\%)$ \\
\hline Age at time of IPAA, y & 27.5 & \\
Sex, female:male & $(10.2-58.5)$ & \\
& & $92: 100$ \\
Follow-up since IPAA, y & 10.1 & $(47.9: 52.1)$ \\
No. of follow-up endoscopies & $(5.5-15.4)$ & \\
No. of bowel movements & $8(5-11)$ & \\
Pouch adenomas & $6(5-8)$ & \\
Duodenal adenomas & & $90(46.9)$ \\
Gastric adenomas & & $147(80.3)$ \\
Desmoid tumors & & $68(37.2)$ \\
\hline
\end{tabular}

Results of quantitative and categorical variables are presented as median with interquartile range (IQR) unless otherwise stated and as absolute and relative frequencies.

27.5 years (range, 10.2-58.5 y; Table 1 ). The median number of follow-up endoscopies was 8 (IQR, 5-11).

Histologically confirmed adenomas arising from the pouch mucosa were detected in 90 patients $(46.9 \%)$ at a median of 8.5 years (range, $0.9-25.1 \mathrm{y}$ ) after IPAA. Among them, 46 patients $(51.1 \%)$ had $<4,14(15.6 \%)$ had 5 to 10 , and $30(33.3 \%)$ had $>10$ adenomas within the pouch. The maximum size of the pouch adenomas was $\leq 4 \mathrm{~mm}$ in 53 patients $(58.9 \%), 5$ to $10 \mathrm{~mm}$ in 24 patients $(26.7 \%)$, and $>10 \mathrm{~mm}$ in 13 patients $(14.4 \%)$. Tubular adenomas were detected in 69 patients $(76.7 \%)$, tubulovillous adenomas were detected in 16 patients (17.8\%), and villous adenomas were detected in 5 patients (5.6\%). Thirty-two patients (35.9\%) showed progression of pouch adenomas during follow-up.

Upper-GI endoscopy reports were available for 183 patients. Gastric adenomas were histologically verified in $68(37.2 \%)$ of 183 patients, and duodenal adenomas were verified in 147 patients $(80.3 \%)$. Forty-seven patients $(24.5 \%)$ experienced desmoid tumors, whereas $36(18.6 \%)$ had neither pouch adenomas nor duodenal or gastric adenomas. None of the patients developed pouch adenomas before closure of their ileostomy. One patient developed a pouch carcinoma 27 years after IPAA, necessitating pouch extirpation and reconstruction of a new pouch.

\section{Follow-up Interval, Age, and Sex}

Age at the time of IPAA did not differ significantly between patients with and without pouch adenomas $(p=0.1901)$. However, the follow-up period and the duration of proper pouch function were significantly longer among patients with pouch adenomas than those without pouch adenomas $(p<0.0001$; Table 2$)$. In addition, significantly more male patients developed pouch adenomas $(p=0.0083$; Table 2$)$.

\section{Extracolonic Manifestations}

Patients with gastric $(\mathrm{n}=68 ; p=0.0004)$ or duodenal adenomas ( $n=147 ; p=0.0014)$ developed pouch adenomas significantly more frequently than those without gastric or duodenal adenomas (Table 2). The presence of desmoid tumors, however, was not related to the occurrence of pouch adenomas $(p=0.5078$; Table 2$)$.

\section{Number of Bowel Movements and Pouchitis}

The median number of bowel movements was 6 (IQR, $5-8)$ per 24 hours. The number of bowel movements per 24 hours did not differ between patients with and without pouch adenomas $(p=0.9734)$. Histologically proven pouchitis persisting for $>3$ months was found in $31.8 \%$ of the patients but did not have an impact on the development of pouch adenomas $(p=0.6622$; Table 2$)$.

\section{Mutation Analysis}

Information on the underlying germline mutation was available for 66 patients $(73.3 \%)$ with pouch adenomas and 67 patients $(65.7 \%)$ without pouch adenomas. Most

TABLE 2. Demographic data of patients with and without pouch adenomas

\begin{tabular}{|c|c|c|c|}
\hline Total & $\begin{array}{c}\text { Presence of pouch } \\
\text { adenomas, } N=90 \text { (46.9\%) }\end{array}$ & $\begin{array}{c}\text { Absence of pouch } \\
\text { adenomas, } N=102(53.1 \%)\end{array}$ & $p$ \\
\hline Age at time of IPAA, mean (SD), y & $27.8(9.4)$ & $30.6(11.7)$ & 0.1901 \\
\hline \multicolumn{4}{|l|}{ Follow-up since IPAA, y } \\
\hline Mean with SD & $13.9(6.9)$ & $8.1(6.2)$ & \multirow[t]{2}{*}{$<0.0001^{\mathrm{a}}$} \\
\hline Median with IQR & $12.8(9.0-17.0)$ & $7.3(2.5-12.2)$ & \\
\hline \multicolumn{4}{|l|}{ Sex } \\
\hline Male patients $(n=100)$ & $56(56.0)$ & $44(44.0)$ & \multirow[t]{2}{*}{$0.0083^{a}$} \\
\hline Female patients $(n=92)$ & $34(37.0)$ & $58(63.0)$ & \\
\hline No. of bowel movements & $6.8(3.1)$ & $6.8(2.6)$ & 0.9734 \\
\hline $\begin{array}{l}\text { Duodenal adenomas }(n=147) \text { of } 183 \text { patients } \\
\text { with regular gastroscopies }\end{array}$ & $81(55.1)$ & $66(44.9)$ & $0.0014^{\mathrm{a}}$ \\
\hline $\begin{array}{l}\text { Gastric adenomas }(n=68) \text { of } 183 \text { patients } \\
\text { with regular gastroscopies }\end{array}$ & $45(66.2)$ & $23(33.8)$ & $0.0004^{\mathrm{a}}$ \\
\hline Desmoid tumors $(n=47)$ & $24(51.1)$ & $23(48.9)$ & 0.5078 \\
\hline Pouchitis $(n=61)$ (histological signs for $>3$ mo) & $30(49.2)$ & $31(50.8)$ & 0.6622 \\
\hline
\end{tabular}


patients in both groups had mutations within exon 15 of $A P C$, primarily at the $5^{\prime}$ end of codon 1309 . No difference in the distribution of germline mutations was found between patients with and without pouch adenomas or between male and female patients with pouch adenomas.

\section{Pouch Adenoma-Free Survival}

Kaplan-Meier estimation of pouch adenoma-free survival shows that, 5 years after IPAA, $84.9 \%$ of patients remain free of pouch adenomas. After 15 years, however, only $40.4 \%$ remain free of pouch adenomas, and after 20 years only $21.9 \%$ of the patients will not have developed pouch adenomas (Fig. 1A). Patients who underwent IPAA at $\leq 18$ years of age developed pouch adenomas significantly earlier than those $>19$ years at the time of their IPAA $(p=0.0173)$. Likewise, patients with gastric adenomas $(p=0.0019)$ and males $(p=0.0017)$ showed pouch adenomas significantly

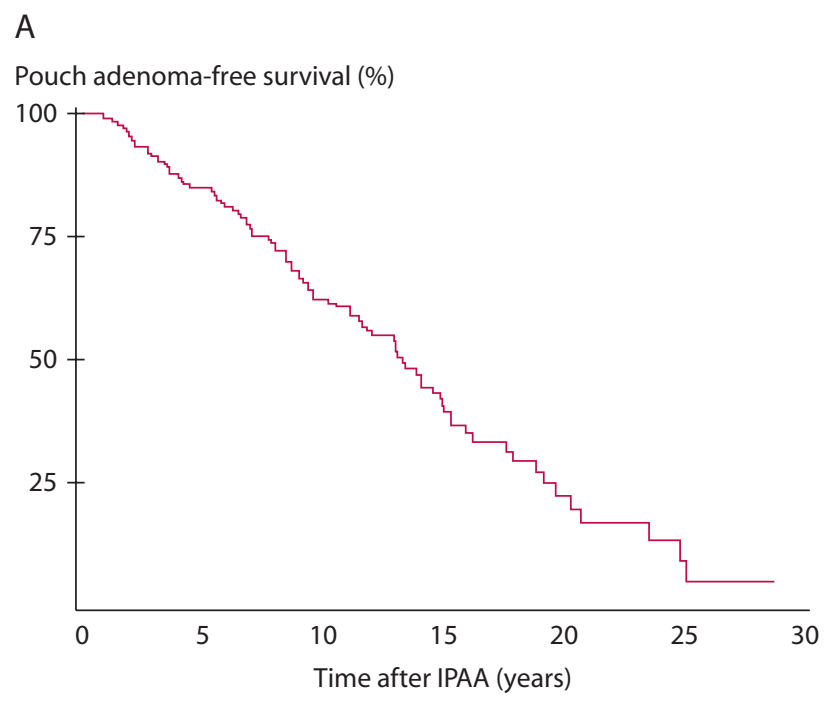

C

Pouch adenoma-free survival (\%)

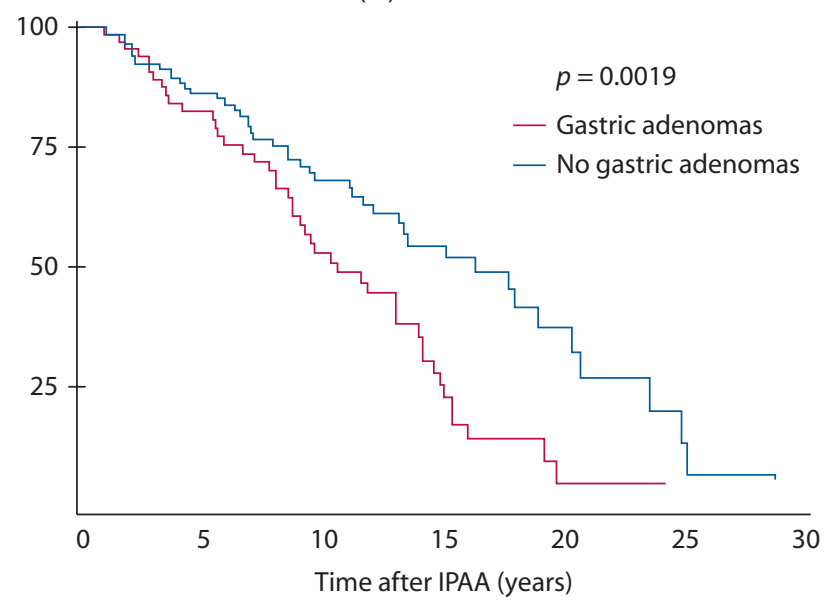

earlier than others (Figs. 1B-D). Estimation of pouch adenoma-free survival in patients with or without duodenal adenomas suggests earlier development of pouch adenomas in the presence of duodenal adenomas, although the correlation was not significant $(p=0.1462)$. Multivariate Cox regression analysis confirmed that male sex $(\mathrm{HR}=2.4(95 \%$ CI, 1.5-3.8)), the presence of gastric adenomas $(\mathrm{HR}=1.8$ $(95 \% \mathrm{CI}, 1.2-2.8)$ ), and age at the time of IPAA $\leq 18$ years $(\mathrm{HR}=2.5(95 \% \mathrm{CI}, 1.4-4.3))$ were independently associated with a significantly higher risk for developing pouch adenomas $(p=0.0002, p=0.0059$, and $p=0.0020$ ).

\section{DISCUSSION}

To our knowledge, the present study includes the largest cohort of patients with FAP who has been screened for a prevalence of pouch adenomas and their underlying risk

\section{B}

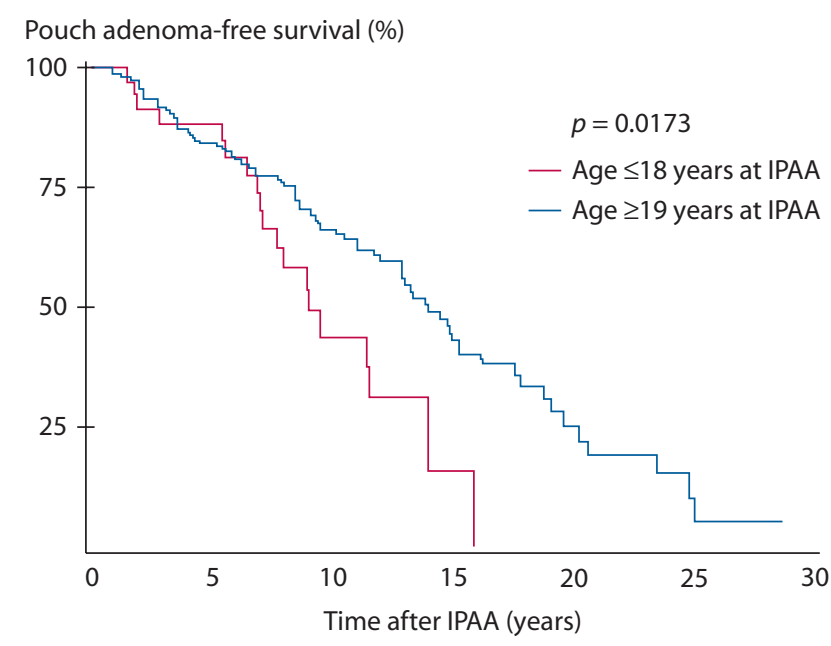

D

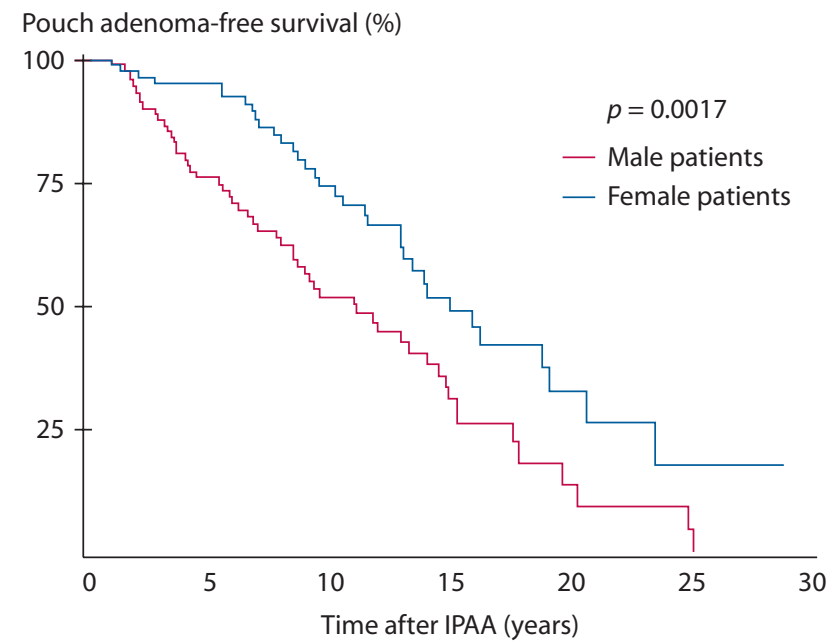

FIGURE 1. A, Kaplan-Meier curve showing overall pouch adenoma-free survival. B, Kaplan-Meier curves comparing pouch adenoma-free survival between patients $\leq 18$ and $\geq 19$ years of age at the time of IPAA. C, Kaplan-Meier curves comparing pouch adenoma-free survival between patients with and without gastric adenomas. D, Kaplan-Meier curves comparing pouch adenoma-free survival between male and female patients. 
factors during follow-up after IPAA. Given that nearly half of the study participants developed pouch adenomas during the follow-up period, we would anticipate that only $\approx 22 \%$ of patients would be free of pouch adenomas 20 years after surgery. Pouch adenomas thus appear to represent a highly relevant risk for patients with FAP. The prevalence of pouch adenomas in the present study is consistent with earlier reported pouch adenoma rates in smaller FAP populations. ${ }^{6-9}$

This present study revealed 3 main independent risk factors for the development of pouch adenomas: age $<18$ years at the time of IPAA, male sex, and the presence of gastric adenomas. Although young age at the time of surgery had been reported previously to be a risk factor, male sex and the presence of gastric adenomas had not been identified as predictors for the development of pouch adenomas. ${ }^{9}$ In addition, we have confirmed that the postoperative interval after IPAA is a relevant risk factor for the development of pouch adenomas. ${ }^{5,7-9}$ Consistent with the findings of Parc et al, ${ }^{9}$ the presence of duodenal adenomas was also associated with a higher probability for pouch adenoma development in our series of patients with FAP. Thus, male sex and a severe GI phenotype with a relatively early disease onset are associated with a higher risk of pouch adenoma development. In line with earlier studies, we detected no association between pouch adenomas and the underlying germline mutation for either the whole population or among affected males and females. ${ }^{6,9}$ However, mutation analysis was not available for all of the patients, which certainly limits the strength of this finding.

Our Kaplan-Meier estimation of pouch adenomafree survival over time raises concerns. Although 5 years after IPAA $\approx 85 \%$ of patients were free of pouch adenomas, this proportion is expected to decline to barely $22 \%$ within 20 years after IPAA. These findings are consistent with the estimated $72 \%$ risk of pouch adenoma development after 20 years reported by Tajika et $\mathrm{al}^{5}$ and the $75 \%$ risk after 15 years reported by Parc et al..$^{9}$ This implies that a number of adenoma-free patients in the present study will likely be affected by pouch adenomas in the future. Considering that most patients are treated surgically in their third decade of life and that the ileal pouch will likely be functioning for $>20$ years, the vast majority of patients with FAP will develop pouch adenomas with the possible potential for malignant transformation. This means that close surveillance of the pouch is mandatory, and new guidelines for the management of pouch adenomas are essential. Moreover, in cases where rectal involvement is absent or only mild, colectomy with ileorectal anastomosis should be considered to prolong the interval until pouch surgery becomes inevitable.

Groves et $\mathrm{al}^{8}$ suggested an algorithm for the management of pouch adenomas. They recommended endoscopic resection in cases of moderate dysplasia or villous architecture. The feasibility and effectiveness of endoscopic resection of pouch adenomas should be thoroughly evaluated, especially in patients at high risk, as identified in the present study. In cases where the safety of endoscopic surveillance is reduced by severe pouch adenomatosis, prophylactic pouch removal to avoid malignant transformation must be discussed, possibly with the chance of new pouch reconstruction.

Based on our present findings, we recommend use of a standardized follow-up protocol and documentation to reduce interphysician differences. Pouch endoscopy should be performed on a yearly basis and include standardized biopsies if pouch adenomas are observed. In the case of small adenomas, they should be histologically confirmed and followed up, depending on their size. For adenomas $>5 \mathrm{~mm}$, we recommend endoscopic removal. If a single high-grade dysplasia is diagnosed, an endoscopic mucosectomy should be performed, if possible. In cases with multiple adenomas causing a difficult surveillance situation or detection of high-grade dysplasia, pouch removal and possible reconstruction must be discussed.

\section{CONCLUSION}

Pouch adenomas affect the vast majority of patients with FAP after IPAA. A severe upper intestinal phenotype, male sex, and age $<18$ years at the time of IPAA all increase the risk of developing pouch adenomas. Thorough endoscopic surveillance of the pouch is mandatory for patients with FAP, even more so if they carry the aforementioned risk factors. Guidelines for the management of pouch adenomatosis are needed, including an algorithm with therapeutic options taking into consideration pouch adenoma severity.

\section{REFERENCES}

1. Fazio VW, O’Riordain MG, Lavery IC, et al. Long-term functional outcome and quality of life after stapled restorative proctocolectomy. Ann Surg. 1999;230:575-584.

2. Ganschow P, Pfeiffer U, Hinz U, Leowardi C, Herfarth C, Kadmon M. Quality of life ten and more years after restorative proctocolectomy for patients with familial adenomatous polyposis coli. Dis Colon Rectum. 2010;53:1381-1387.

3. Osterfeld N, Kadmon M, Brechtel A, Keller M. Preoperative and postoperative quality of life in patients with familial adenomatous polyposis. Dis Colon Rectum. 2008;51:1324-1330.

4. Bülow $\mathrm{S}$. Results of national registration of familial adenomatous polyposis. Gut. 2003;52:742-746.

5. Tajika M, Nakamura T, Nakahara O, et al. Prevalence of adenomas and carcinomas in the ileal pouch after proctocolectomy in patients with familial adenomatous polyposis. J Gastrointest Surg. 2009; 13:1266-1273.

6. Tonelli F, Ficari F, Bargellini T, Valanzano R. Ileal pouch adenomas and carcinomas after restorative proctocolectomy for familial adenomatous polyposis. Dis Colon Rectum. 2012; 55:322-329. 
7. Wu JS, McGannon EA, Church JM. Incidence of neoplastic polyps in the ileal pouch of patients with familial adenomatous polyposis after restorative proctocolectomy. Dis Colon Rectum. 1998;41:552-556.

8. Groves CJ, Beveridge lG, Swain DJ, et al. Prevalence and morphology of pouch and ileal adenomas in familial adenomatous polyposis. Dis Colon Rectum. 2005;48:816-823.

9. Parc YR, Olschwang S, Desaint B, Schmitt G, Parc RG, Tiret E. Familial adenomatous polyposis: prevalence of adenomas in the ileal pouch after restorative proctocolectomy. Ann Surg. 2001;233:360-364.
10. Pommaret E, Vienne A, Lefevre JH, et al. Prevalence and risk factors for adenomas in the ileal pouch and the afferent loop after restorative proctocolectomy for patients with familial adenomatous polyposis. Surg Endosc. 2013;27: 3816-3822.

11. Zahid A, Kumar S, Koorey D, Young CJ. Pouch adenomas in familial adenomatous polyposis after restorative proctocolectomy. Int J Surg. 2015;13:133-136.

12. Spigelman AD, Williams CB, Talbot IC, Domizio P, Phillips RK. Upper gastrointestinal cancer in patients with familial adenomatous polyposis. Lancet. 1989;2:783-785. 